\title{
Modeling HIV-1 Viral Capsid Nucleation by Dynamical Systems
}

\author{
Farrah Sadre-Marandi * ${ }^{*} \quad$ Yuewu Liu ${ }^{\dagger}$ Jiangguo Liu ${ }^{\ddagger}$

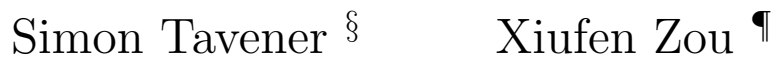

September 7, 2015

\begin{abstract}
There are two stages generally recognized in the viral capsid assembly: nucleation and elongation. This paper focuses on the nucleation stage and develops mathematical models for HIV-1 viral capsid nucleation based on six-species dynamical systems. The Particle Swarm Optimization (PSO) algorithm is used for parameter fitting to estimate the association and dissociation rates from biological experiment data. Numerical simulations of capsid protein (CA) multimer concentrations demonstrate a good agreement with experimental data. Sensitivity and elasticity analysis of CA multimer concentrations with respect to the association and dissociation rates further reveals the importance of CA trimer-of-dimers in the nucleation stage of viral capsid self-assembly.
\end{abstract}

Keywords: capsid, dimers, dynamical systems, hexamers, HIV-1, pentamers, parameter fitting, sensitivity analysis

\section{Introduction}

Viruses are macromolecular organisms that are composed of infective genetic materials (DNA or RNA) and protective protein shells. Understanding the mechanism in

*Department of Mathematics, Colorado State University, Fort Collins, CO 80523-1874, USA, sadre@math. colostate.edu

†School of Mathematics and Statistics, Wuhan University, Wuhan 430072, Hubei, China, yuewuliu@whu.edu.cn

${ }_{\ddagger}^{\ddagger}$ Department of Mathematics, Colorado State University, Fort Collins, CO 80523-1874, USA, liu@math. colostate.edu

${ }^{\S}$ Department of Mathematics, Colorado State University, Fort Collins, CO 80523-1874, USA, tavener@math. colostate.edu

'School of Mathematics and Statistics, Wuhan University, Wuhan 430072, Hubei, China, xzou@whu.edu.cn 
the viral life cycle, particularly the entry, replication, egress, and capsid assembly will be helpful for developing effective treatments of viral diseases. While in vivo and in vitro approaches offers direct ways for investigating all stages of the viral life cycle, the in silico approach (mathematical modeling and computer simulations) plays an increasingly important role in virus studies. Molecular dynamics (MD) is a powerful tool for simulating viral capsid assembly [44] but places high demand on computing resources, although coarse-grain (CG) models, e.g., [10], can reduce the computational cost. In this paper, we explore an inexpensive approach based on the rate equations and dynamical systems.

Dynamical systems or systems of ordinary differential equations have been used for modeling the replication and pathogenesis of human immunodeficiency virus (HIV) [42], HIV virus dynamics [21, 31] and infection dynamics of other types viruses [24], including sensitivity analysis of system behaviors to model parameters. But in this paper, we use dynamical systems for modeling the structural biological aspects of HIV. We perform also sensitivity and elasticity analysis for our models. This is a continuation of our efforts in $[19,35,36,37,41]$ on applying dynamical systems and/or sensitivity analysis as mathematical tools for investigation of biological processes.

HIV-1 is a retrovirus that causes acquired immunodeficiency syndrome (AIDS), a condition in humans in which the immune system fails progressively. It is known that the HIV-1 virion undergoes a maturation process, in which the viral RNA is enclosed by a cone-shape capsid so that the virion becomes infectious. The HIV-1 viral capsid assembly consists of two stages: nucleation and elongation. Understanding the mechanism in the viral capsid assembly is important and will be helpful for developing antiviral therapies that could target viral capsids.

Structural biology research of the HIV-1 virus indicates that HIV-1 conical cores have a lattice structure consisting of hexamers and pentamers. At the early stage of viral capsid assembly, lower order CA proteins nucleate into hexamers. These hexamers further assemble into the viral capsid. There have been kinetic models for viral capsid assembly $[12,18]$. But these models consider a simplified pathway that allows association or dissociation of one capsomer unit at a time. However, there is strong evidence $[8,10,16]$ that dimers associate with other dimers. Moreover, non-monomer subunits can assemble with each other [28, 30].

In this paper, we focus on the nucleation stage of viral capsid assembly but consider nearly all possible pathways of association and dissociation. In particular, we develop mathematical models for nucleation using dynamical systems of six species. The biological evidence $[7,10,16,25,43]$ are then used to reduce the model. Published biological experimental data [30] are utilized to estimate the model parameters representing the association and dissociation rates. Furthermore, sensitivity and elasticity analysis is performed to find out what association / dissociation terms play more important roles in the nucleation stage. 
The rest of this paper is organized as follows. Section 2 presents first a full 6species model for nucleation kinetics and then a reduced 6-species model. Section 3 discusses the methods for model parameter fitting and sensitivity analysis and elasticity analysis. Section 4 presents results of numerical simulations of CA multimer concentrations along with sensitivity and elasticity analysis. Section 5 concludes the paper with discussion for future work.

\section{Models for CA Protein Nucleation}

The existing work in $[12,18,26]$ adopt a straightforward approach by considering one pathway of assembly: only one CA protein (monomer) can assemble with another subunit at a time, that is, from $n$-mer to $(n+1)$-mer. Similarly, the dissociation is from $(n+1)$-mer to $n$-mer. However, there is strong evidence $[8,10,16]$ that dimers interact with other dimers. The findings in $[28,30]$ suggest that non-monomer subunits can assemble with each other. Stability analysis in [10] indicates that the dimer is an important CA intermediate in self-assembly.

Based on the aforementioned work, we start with a new model by considering all possible pathways for forming a nucleus, also referred to as a hexamer or 6-mer. We follow the traditional model for polymer growth, which states that any two intermediates can react and join together [33]. Additionally, we add a pathway (b) to mimic the trimer-of-dimers assembly discussed in $[7,10,16]$. Since dissociation is also important, due to high concentrations of intermediates left after nucleation, more terms are added to describe the multitude of dissociations. We assume that multimers can dissociate in the same way in which they are formed during association.

\subsection{A Full 6-species Model}

Listed below are the assumptions for our 6-species model.

1. Nucleation ends with 6-mer formation. [28, 30] observed little to no existence of $c_{n}, n>6$.

2. One forward rate for each intermediate.

3. Multimers can dissociate in the same way they are formed in association.

Based on the above assumptions, a dynamical system of size 6 or a system of six ordinary differential equations is proposed as follows for describing the kinetics in the 
association and dissociation.

$$
\left\{\begin{aligned}
\frac{d c_{1}}{d t}= & b_{65} c_{6}+b_{54} c_{5}+b_{43} c_{4}+b_{32} c_{3}+2 b_{21} c_{2} \\
& -f_{15} c_{1} c_{5}-f_{14} c_{1} c_{4}-f_{13} c_{1} c_{3}-f_{12} c_{1} c_{2}-2 f_{11} c_{1}^{2} \\
\frac{d c_{2}}{d t}= & f_{11} c_{1}^{2}+3 b_{62} c_{6}+b_{64} c_{6}+b_{53} c_{5}+2 b_{42} c_{4}+b_{32} c_{3} \\
& -b_{21} c_{2}-3 f_{222} c_{2}^{3}-f_{24} c_{2} c_{4}-f_{23} c_{2} c_{3}-2 f_{22} c_{2}^{2}-f_{12} c_{1} c_{2} \\
\frac{d c_{3}}{d t}= & f_{12} c_{1} c_{2}+2 b_{63} c_{6}+b_{53} c_{5}+b_{43} c_{4}-b_{32} c_{3}-2 f_{33} c_{3}^{2}-f_{23} c_{2} c_{3}-f_{13} c_{1} c_{3} \\
\frac{d c_{4}}{d t}= & f_{13} c_{1} c_{3}+f_{22} c_{2}^{2}+b_{64} c_{6}+b_{54} c_{5}-b_{43} c_{4}-b_{42} c_{4}-f_{24} c_{2} c_{4}-f_{14} c_{1} c_{4} \\
\frac{d c_{5}}{d t}= & f_{14} c_{1} c_{4}+f_{23} c_{2} c_{3}+b_{65} c_{6}-b_{54} c_{5}-b_{53} c_{5}-f_{15} c_{1} c_{5} \\
\frac{d c_{6}}{d t}= & f_{15} c_{1} c_{5}+f_{33} c_{3}^{2}+f_{222} c_{2}^{3}+f_{24} c_{2} c_{4}-b_{65} c_{6}-b_{64} c_{6}-b_{63} c_{6}-b_{62} c_{6}
\end{aligned}\right.
$$

where

- $c_{n}$ is the concentration of the $n$-mer intermediate $(1 \leq n \leq 6)$;

- $f_{i j}$ is the association rate of $c_{i}$ and $c_{j}$;

- $f_{222}$ is the association rate for trimer-of-dimer;

- $b_{i j}$ is the rate of $c_{i}$ dissociating into two intermediates with $c_{j}$ being the larger intermediate of the dissociated terms, $b_{62}$ is for the special case 6 -mer dissociates into three dimers.



Figure 1: A diagram for the second pathway (trimer-of-dimers) of hexamer assembly. Protein illustrations are drawn according to the info about PDB 3H47 HIV-1 CA monomer shown in [29] and used by [30]. 


\subsection{A Reduced 6-species Model}

The above full 6-species model considers all possible pathways of two binding intermediates and one triple bond in the association leading to and dissociation down from hexamers. We simplify this model based on the findings in the literature about viral capsid assembly.

1. We consider three main pathways for assembly of a hexamer:

(a) Single monomers join:

$$
c_{1}+c_{1} \underset{b_{21}}{\stackrel{f_{11}}{\rightleftharpoons}} c_{2}, \quad c_{1}+c_{2} \underset{b_{32}}{\stackrel{f_{12}}{\rightleftharpoons}} c_{3}, \quad c_{1}+c_{3} \underset{b_{43}}{\stackrel{f_{13}}{\rightleftharpoons}} c_{4}, \quad c_{1}+c_{4} \underset{b_{54}}{\stackrel{f_{14}}{\rightleftharpoons}} c_{5}, \quad c_{1}+c_{5} \underset{b_{65}}{\stackrel{f_{15}}{\rightleftharpoons}} c_{6} .
$$

(b) Trimer-of-dimers as illustrated in Figure 1:

$$
c_{1}+c_{1} \underset{b_{21}}{\stackrel{f_{11}}{\rightleftharpoons}} c_{2}, \quad c_{2}+c_{2}+c_{2} \underset{b_{62}}{\stackrel{f_{222}}{\rightleftharpoons}} c_{6} .
$$

(c) Single binding dimers:

$$
c_{1}+c_{1} \underset{b_{21}}{\stackrel{f_{11}}{\rightleftharpoons}} c_{2}, \quad c_{2}+c_{2} \underset{b_{42}}{\stackrel{f_{22}}{\rightleftharpoons}} c_{4}, \quad c_{2}+c_{4} \underset{b_{64}}{\stackrel{f_{24}}{\rightleftharpoons}} c_{6} .
$$

2. We consider two pathways for formation of a pentamer:

(d) Single monomers join (viewed as a part of pathway (a) for hexamers:

$$
c_{1}+c_{1} \underset{b_{21}}{\stackrel{f_{11}}{\rightleftharpoons}} c_{2}, \quad c_{1}+c_{2} \underset{b_{32}}{\stackrel{f_{12}}{\rightleftharpoons}} c_{3}, \quad c_{1}+c_{3} \underset{b_{43}}{\stackrel{f_{13}}{\rightleftharpoons}} c_{4}, \quad c_{1}+c_{4} \underset{b_{54}}{\stackrel{f_{14}}{\rightleftharpoons}} c_{5} .
$$

(e) Dimers and monomers:

$$
c_{1}+c_{1} \underset{b_{21}}{\stackrel{f_{11}}{\rightleftharpoons}} c_{2}, \quad c_{2}+c_{2} \underset{b_{42}}{\stackrel{f_{22}}{\rightleftharpoons}} c_{4}, \quad c_{1}+c_{4} \underset{b_{54}}{\stackrel{f_{14}}{\rightleftharpoons}} c_{5} .
$$

It is important to note that the two pathways for formation of a pentamer (d) and (e) do not add new forward or backward rates to the model. Pathway (d) is a subset of pathway (a) for a hexamer, and pathway (e) is a combination of the intermediate pathways found in (a) and (b).

The hexamer pathways are based on the findings presented in [25]. The first pathway (a) (monomers join one at a time) was adopted in $[12,18,26]$. "The symmetric appearance (of a hexamer) is suggestive of symmetric head-to-head dimers" promoting the trimer-of-dimer assembly seen in the second pathway (b), see Figure 1. This is also advocated in $[7,10,16]$. The third pathway (c) for a hexamer considered 
in our reduced model is established based on the discussion in $[4,8,15,22,23,25,40]$. In particular, [40] asserts that CA prefers to form both dimers and tetramers. This pathway could also be considered as the "slow" formation of trimer-of-dimers. Considering only these three pathways eliminates the parameter $f_{33}$ and the corresponding backward rate $b_{63}$ from the model.

The pentamer pathways are also listed, since pentamers are required for formation of a closed viral capsid [3, 5, 13, 27]. Both pathways for pentamer formation occur as either a subpathway or union of hexamer pathways. Note that considering only these two pathways for pentamers allows the elimination of the term $f_{23} c_{2} c_{3}$, and its corresponding dissociation term $b_{53} c_{5}$ from the full model.

Consideration of these pathways reduces emphasis on trimers. Even though trimers of matrix proteins (MA) are predominately observed during the assembly of immature virions $[4,41]$, there is not much evidence that the CA proteins prefers trimer formation [1].

The above discussion leads to a reduced 6-species model:

$$
\left\{\begin{aligned}
\frac{d c_{1}}{d t}= & b_{65} c_{6}+b_{54} c_{5}+b_{43} c_{4}+b_{32} c_{3}+2 b_{21} c_{2} \\
& -f_{15} c_{1} c_{5}-f_{14} c_{1} c_{4}-f_{13} c_{1} c_{3}-f_{12} c_{1} c_{2}-2 f_{11} c_{1}^{2} \\
\frac{d c_{2}}{d t}= & f_{11} c_{1}^{2}+3 b_{62} c_{6}+b_{64} c_{6}+2 b_{42} c_{4}+b_{32} c_{3} \\
& -b_{21} c_{2}-3 f_{222} c_{2}^{3}-f_{24} c_{2} c_{4}-2 f_{22} c_{2}^{2}-f_{12} c_{1} c_{2} \\
\frac{d c_{3}}{d t}= & f_{12} c_{1} c_{2}+b_{43} c_{4}-b_{32} c_{3}-f_{13} c_{1} c_{3} \\
\frac{d c_{4}}{d t}= & f_{13} c_{1} c_{3}+f_{22} c_{2}^{2}+b_{64} c_{6}+b_{54} c_{5}-b_{43} c_{4}-b_{42} c_{4}-f_{24} c_{2} c_{4}-f_{14} c_{1} c_{4} \\
\frac{d c_{5}}{d t}= & f_{14} c_{1} c_{4}+b_{65} c_{6}-b_{54} c_{5}-f_{15} c_{1} c_{5} \\
\frac{d c_{6}}{d t}= & f_{15} c_{1} c_{5}+f_{222} c_{2}^{3}+f_{24} c_{2} c_{4}-b_{65} c_{6}-b_{64} c_{6}-b_{62} c_{6}
\end{aligned}\right.
$$

where $c_{n}, f_{i j}$, and $b_{i j}$ bear the same meaning as described in the full 6 -species model.

This reduced 6-species model will be used for numerical simulations of CA protein nucleation. Sensitivity and elasticity of the intermediate concentrations $c_{n}(n=$ $1, \ldots, 6)$ to the forward and backward rates will be analyzed also (See the Section "Results"). 


\section{Materials and Methods}

\subsection{An Optimization Algorithm for Model Parameter Fitting}

To obtain values of the model parameters based on published experimental data, we adopt the Particle Swarm Optimization (PSO) method [11]. PSO is a method for optimizing continuous nonlinear functions. PSO has an open-source Matlab implementation, which will be used in this paper to optimize the values of the sixteen parameters in the reduced model for viral capsid nucleation under certain constraints on the forward and backward rates.

PSO is a numerical method based on the stochastic optimization technique developed by Eberhart and Kennedy [11] in 1995. Since then, it has been widely used in many research fields, for example, neural network, telecommunications, design, control, signal processing, power systems, and data mining.

PSO optimizes a problem by having a population of candidate solutions (particles). It tries iteratively to improve the solutions with regard to additional constraints by updating generations until the target is met. In each iteration, the solutions are updated by tracking two values: one is the best solution or fitness (p) each parameter has achieved, the other is the best value obtained by any other particle in the population $(g \mathbf{1})$.

After finding the two best values up to that time, the solutions update their velocities and positions by the following formulas:

$$
\begin{aligned}
\mathbf{v}(i+1) & =w \mathbf{v}(i)+d_{1} r_{1}[\mathbf{p}(i)-\mathbf{x}(i)]+d_{2} r_{2}[g \mathbf{1}(i)-\mathbf{x}(i)] \\
\mathbf{x}(i+1) & =\mathbf{x}(i)+\mathbf{v}(i+1)
\end{aligned}
$$

where

- $w$ is the initial inertia weight with a default value 0.9 ;

- $\mathbf{v}(i)$ is the particle velocity at iteration $i$;

- $d_{1}, d_{2}$ are the local best influence and global best influence weights, respectively, typically set to $d_{1}=d_{2}=2$;

- $r_{1}, r_{2}$ are random variables between $(0,1)$;

- $\mathbf{x}(i)$ is the particle position at iteration $i$

- $\mathbf{p}, g \mathbf{1}$ are defined as stated before.

A pseudo code for the procedure is shown as follows. 


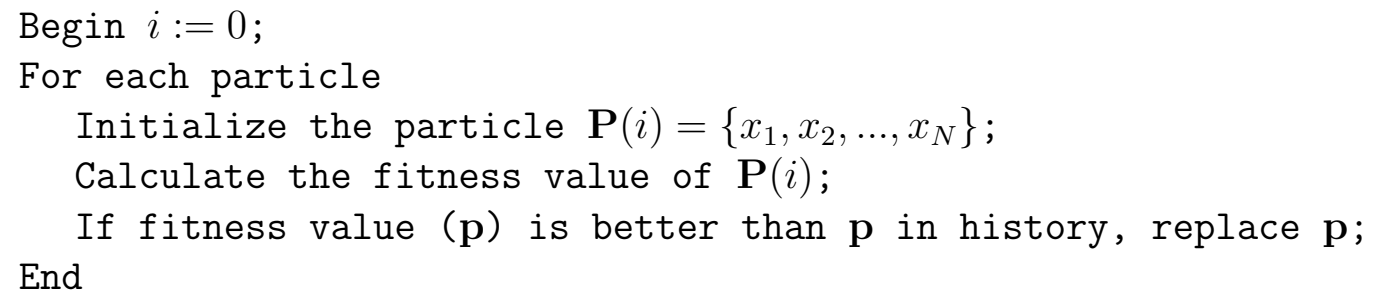

End

\subsection{Constraints on the Forward and Backward Rates}

Before using the PSO algorithm to optimize the parameters, an initial guess $P(1)$ must be chosen. The choice of PSO parameters can have a big impact on optimization performance. The following size order relations on the forward and backward rates help find a good initial guess and set bounds for each parameter.

\subsubsection{Constraints on the Forward Rates}

The models presented in $[12,20,45]$ assume that only one protein is added (could associate) at a time and all forward rates are equivalent. In [26], it is assumed $f_{n}$ (equivalent to $f_{1 n}$ in our model) increases monotonically with $n$. In [28], it is found that monomers assemble spontaneously into a hexamer lattice tube, indicating that the CA proteins tend to form hexamers. Based on these studies, we assume that the forward rates $f_{1 n}$ increases with $n$.

It is expected that $f_{11}$ is very small, since the subunit-subunit interactions are inherently weak $[20,43]$. The pentamer subunit is the least stable intermediate, so $f_{15}$ will be relatively large compared to the others [43].

We adopt a similar size order relation as seen in [26]:

$$
f_{11} \leq f_{12} \ll f_{15} .
$$

[43] discusses the stability of intermediates and claims that a hexamer is more stable than a tetramer and a tetramer is more stable than a pentamer. We assume that stability helps drive intermediate formation and accordingly

$$
f_{22} \leq f_{24} \ll f_{15} .
$$


For the reduced nucleation model presented in this paper, all the forward rates except $f_{222}$ have the physical dimension $T^{-1} L^{3} M^{-1}$, where $T$ is time, $L$ is length, and $M$ is mass. The forward rate $f_{222}$ (for trimer-of-dimer) is the only rate that has a physical dimension $T^{-1}\left(L^{3} M^{-1}\right)^{2}$. It cannot be simply compared to the other forward rates. [10] notes that the trimer-of-dimers structure is crucial for lattice formation, and $[8,16]$ found hexameter formation occurs with increased CA dimer concentration, so it is expected $f_{222}$ to be large.

\subsubsection{Constraints on Backward Rates}

All the backward rates have the physical dimension $T^{-1}$.

The discussion in $[8,10,16]$ implies that it is less likely for a dimer to dissociate. Hence we assume that $b_{21}$ will be the smallest backward rate. Additionally, the instability of pentamers [43] implies that $b_{65}$ should be low compared to that of other hexamer dissociations. These lead to the following assumptions

$$
\begin{aligned}
& b_{21} \leq b_{65} \leq b_{64}, \\
& b_{21} \leq b_{65} \leq b_{62} .
\end{aligned}
$$

\subsection{Sensitivity and Elasticity Analysis}

Sensitivity analysis examines how a system responds to the changes in its parameters. Sensitivity analysis is useful for identifying important parameters that require additional investigation or insignificant parameters that could be eliminated from a model [37, 41].

Sensitivity is computed by finding the derivatives of each variable with respect to each parameter. In other words, the sensitivity of the $i^{\text {th }}$ variable $\left(c_{i}\right)$ with respect to the $k^{t h}$ parameter $\left(p_{k}\right)$ is defined as

$$
S_{i, k}=\frac{\partial c_{i}}{\partial p_{k}}, \quad i=1, \ldots, N, \quad k=1, \ldots, K,
$$

where $N$ is the size of the system and $K$ is the dimension of the parameter space.

Writing a dynamical system as a parametric ODE system

$$
\frac{d c_{i}}{d t}=h_{i}(\mathbf{c}, \mathbf{p}), \quad i=1, \ldots, N ; \quad \mathbf{p} \in \mathbb{R}^{K},
$$

we have the sensitivity of all variables $\left(c_{i}\right)$ with respect to all parameters when the following ODE system is solved:

$$
\frac{d S_{i, k}}{d t}(t)=\left(\sum_{n=1}^{N} \frac{\partial h_{i}}{\partial c_{n}} S_{n, k}(t)\right)+\frac{\partial h_{i}}{\partial p_{k}}(t), \quad S_{i, k}(0)=0 .
$$


However, sensitivity analysis may yield misleading results when the parameter values change greatly in magnitude. Elasticity can produces more reliable results. Elasticity describes the rate of change of the relative size of the variable with respect to the relative size of the parameter. The elasticity of the $i^{\text {th }}$ variable with respect to the $k^{\text {th }}$ parameter is defined as

$$
E_{i, k}(t)=\frac{p_{k}}{c_{i}(t)} \frac{\partial c_{i}}{\partial p_{k}}(t)
$$

SENSAI [38] is a freely available MATLAB package for performing a forward sensitivity and/or elasticity analysis on parametrized systems of nonlinear dynamical systems. SENSAI evaluates the Jacobian

$$
\frac{\partial h_{i}}{\partial c_{n}}, \quad i, n=1, \ldots, N
$$

and the partial derivatives with respect to the parameters

$$
\frac{\partial h_{i}}{\partial p_{k}}, \quad i=1, \ldots, N, \quad k=1, \ldots, K
$$

symbolically using MuPAD, then solves Equation (11) in MATLAB.

\section{Results}

We first describe the data used for comparison for the model presented in this paper. Parameter fitting is performed for the reduced 6-species model with the parameter constraints explained in Section 3.2 so that the solution of the dynamical system closely matches the experimental data reported in [30]. Numerical simulations are performed. Then sensitivity and elasticity of $n$-mer concentrations to parameters are examined.

\subsection{Use of Biological Experimental Data}

It is known from the discussion in $[28,30,43]$ that the structures of CA hexamers are very difficult to obtain because of the weak interactions holding the hexamers together. Instead mutant CA hexamers were utilized for experiments.

[28] compares each mutant hexamer to the HIV-1 CA hexamer given by the Protein Data Bank (PDB) code 3dik. It is found that four mutants assembling into tubes "appeared similar in morphology to the wild-type tubes". Of the four, only two mutants (A14C/E45C in lane 3 and A42C/T54C in lane 9) have enriched 6-mer bands, which is favorable for hexamer bonding to create the full lattice. 
(j)

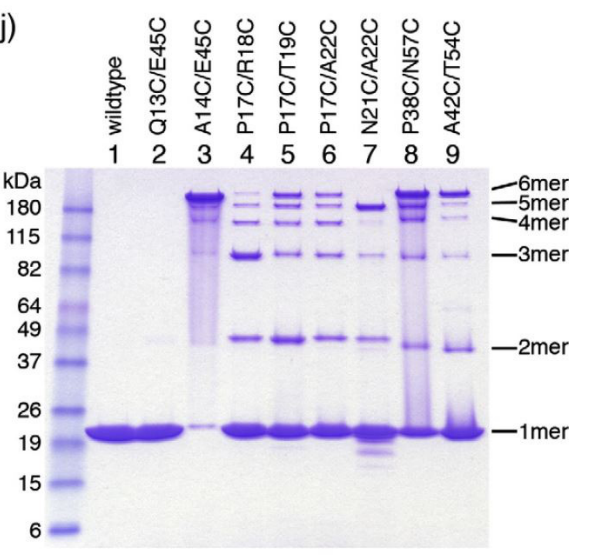



Figure 2: Experimental data of intermediate concentrations. (Left) SDS-PAGE profiles of the assembly. Source: [28] (reprinted with permission from Elsevier). (Right) WT stands for wild type, CC corresponds to A14C/E45C, and CCAA is A14C/E45C/W184A/M185A. Source: [30] (reprinted with permission from Elsevier).

[28] states that A14C/E45C produces hexamers that are the most similar to wildtype HIV-1 hexamers, and adding two more mutations gives the construct A14C/E45C/W184A/M185A even more favorable results. However, no data is reported for this construct.

[30] presents a similar study, creating mutant CA protein that faithfully mimic the hexamer properties of HIV-1 capsid. It is found that the same two mutants A14/E45 and $\mathrm{A} 14 \mathrm{C} / \mathrm{E} 45 \mathrm{C} / \mathrm{W} 184 \mathrm{~A} / \mathrm{M} 185 \mathrm{~A}$ produce the most realistic results. In this case, it is found that the latter mutant assemble less efficiently than A14C/E45C alone.

Both $[28,30]$ consider hexamers stabilized by engineering disulfide cross-link (the mutation) A14/E45 with similar results. [30] gives more information about the protein concentration and timing.

In [30], crosslinked CA A14C/E45C hexamers were prepared by $10 \mathrm{mg} / \mathrm{mL}$ protein into assembly buffer. The buffer is given sequentially, first with $200 \mathrm{mM} \beta$ mercaptoethanol $(\beta \mathrm{ME})$, then $0.2 \mathrm{mM} \beta \mathrm{ME}$, and lastly $20 \mathrm{mM}$ Tris $(\mathrm{pH} 8)$. Each step is performed for 8 hours.

For this paper, we use the data shown in Figure 1 Panel D line 5 in [30] (reprinted in this paper as Figure 2 the Right Panel). In particular, we utilize the image processing software Image J to process the information in the aforementioned image. Each $i$-mer was measured five times to alleviate any discrepancies due to any error occurring in the measuring process. The average of these measurements are used as our ideal equilibrium concentrations. 


\subsection{Results of Model Parameter Fitting}

The initial guess and bounds are constructed using the relationships defined in Section 3.2. PSO is run 10 times due to the randomness involved in Equation (3). Weights are set to the conventional values, with $d_{1}=d_{2}=2$ and $w=0.9$. Iterations are terminated after the max number of iterations $(i=2000)$ or by hitting the minimum global error

$$
|g(i+1)-g(i)|<1 \times 10^{-25}
$$

with a minimum of 250 successive iterations.

We choose the set of parameters that minimize the error between the experimental data and the numerical solution. The optimized parameters yield the lowest relative error (0.0125) are listed in Table 1 . All the forward rates except $f_{222}$ have the physical dimension $T^{-1} L^{3} M^{-1}$, where $T$ is time, $L$ length, and $M$ mass. The forward rate $f_{222}$ has a physical dimension $T^{-1}\left(L^{3} M^{-1}\right)^{2}$. All backward rates have the physical dimension $T^{-1}$. For the numerical simulations in this paper, we use the following units: second for time $T$, millimeter for length $L$, and milligram for mass $M$.

Table 1: Optimal model parameter values used for numerical simulations

\begin{tabular}{|l|l|l|l|}
\hline \hline$f_{11}=0.000556$ & $f_{12}=0.004506$ & $f_{13}=0.000867$ & $f_{14}=0.038226$ \\
\hline$f_{15}=0.179675$ & $f_{22}=0.013196$ & $f_{222}=0.159765$ & $f_{24}=0.061905$ \\
\hline$b_{65}=0.193838$ & $b_{64}=0.256905$ & $b_{62}=0.993826$ & $b_{54}=0.056015$ \\
\hline$b_{43}=0.728455$ & $b_{42}=0.719905$ & $b_{32}=0.717905$ & $b_{21}=0.019094$ \\
\hline
\end{tabular}

\subsection{Results of Multimer Concentrations $\left(c_{1}, c_{2}, c_{3}, c_{4}, c_{5}, c_{6}\right)$}

Now we discuss the stability of equilibria for the reduced 6-species model. First, we reduce the system according to the mass conservation law and our initial condition is $\vec{c}(0)=(1300,0,0,0,0,0)$. This means

$$
c_{1}+2 c_{2}+3 c_{3}+4 c_{4}+5 c_{5}+6 c_{6}=1300 .
$$

The equilibria of the mass-conserving model are found using the solve function in MATLAB. Due to the complexity of the model, the parameters are first set to the optimized parameters (Table 1). Then, each equation in the model is set to zero to be solved for the concentration values. Seventeen solutions were found, out of which six were real-valued, as listed in Table 2 . The negative and imaginary equilibrium points are discarded, since they are not biologically meaningful. This reduces the number of biologically possible equilibria to just one (Line 5 in Table 2). The Jacobian of 
Table 2: Real equilibria for Equation (2) evaluated with parameters defined in Table 1.

\begin{tabular}{|lllll|}
\hline$\left(c_{1}\right.$, & $c_{2}$, & $c_{3}$, & $c_{4}$, & $\left.c_{5}\right)$ \\
\hline \hline$(-6.43 \mathrm{E}+60$, & $4.02 \mathrm{E}+59$, & $4.017 \mathrm{E}+59$, & $6.28 \mathrm{E}+57$, & $-1.93 \mathrm{E}+07)$ \\
\hline$(7.29 \mathrm{E}+20$, & $-1.24 \mathrm{E}+20$, & $-5.13 \mathrm{E}+19$, & $-2.70 \mathrm{E}+17$, & $-6.43 \mathrm{E}+04)$ \\
\hline$(-5.94 \mathrm{E}+10$, & $1.74 \mathrm{E}+10$, & $5.97 \mathrm{E}+09$, & $8.52 \mathrm{E}+07$, & $-1.47 \mathrm{E}+04)$ \\
\hline$(-0.419$, & -8.976, & -53.623, & -55.321, & $891.872)$ \\
\hline$(12.846$, & 6.476, & 17.524, & 18.613, & $10.456)$ \\
\hline$(-360.795$, & 7.256, & 57.058, & -0.787, & $-0.483)$ \\
\hline
\end{tabular}

the system is then computed and evaluated at this equilibrium. The eigenvalues are found to be as follows:

$\lambda_{1}=-3.196, \lambda_{2}=-4.600, \lambda_{3}=-179.051, \lambda_{4}=-0.886-0.342 i, \lambda_{5}=-0.886+0.342 i$.

Since each eigenvalue has a negative real part, the equilibrium shown in Figure 4 is stable.

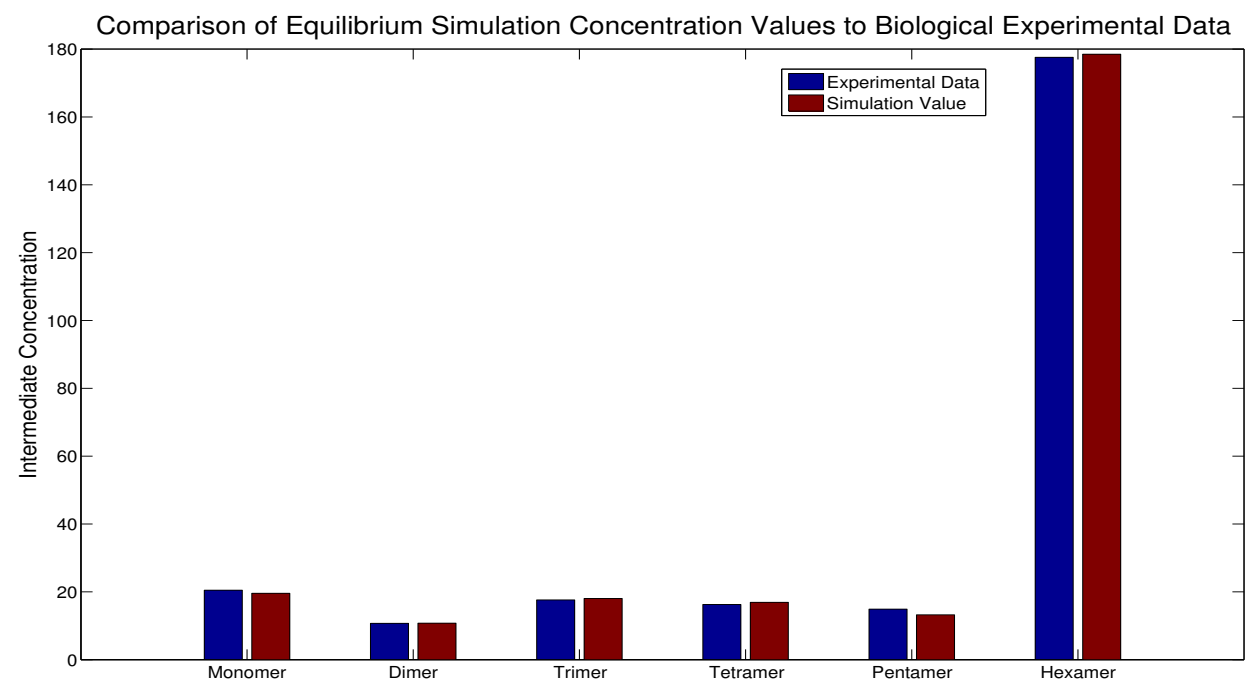

Figure 3: Concentrations of all intermediates $c_{n}(1 \leq n \leq 6)$ at simulation time $t=24 \times 3600$ (second) with initial values $\left(c_{1}(0), c_{2}(0), c_{3}(0), c_{4}(0), c_{5}(0), c_{6}(0)\right)=(1300,0,0,0,0,0)$. The simulation results with optimized model parameters (shown in dark red) demonstrate good agreement with the experimental data in [30], 24 hours after the experiment (shown in dark blue).

The monomer concentration $c_{1}$ quickly decreases as the CA proteins bind with $c_{i}$ concentrations to form $c_{i+1}$ intermediates. Note that there is a large initial spike in the dimer concentration $c_{2}$, implying many monomers bind together to form dimers 

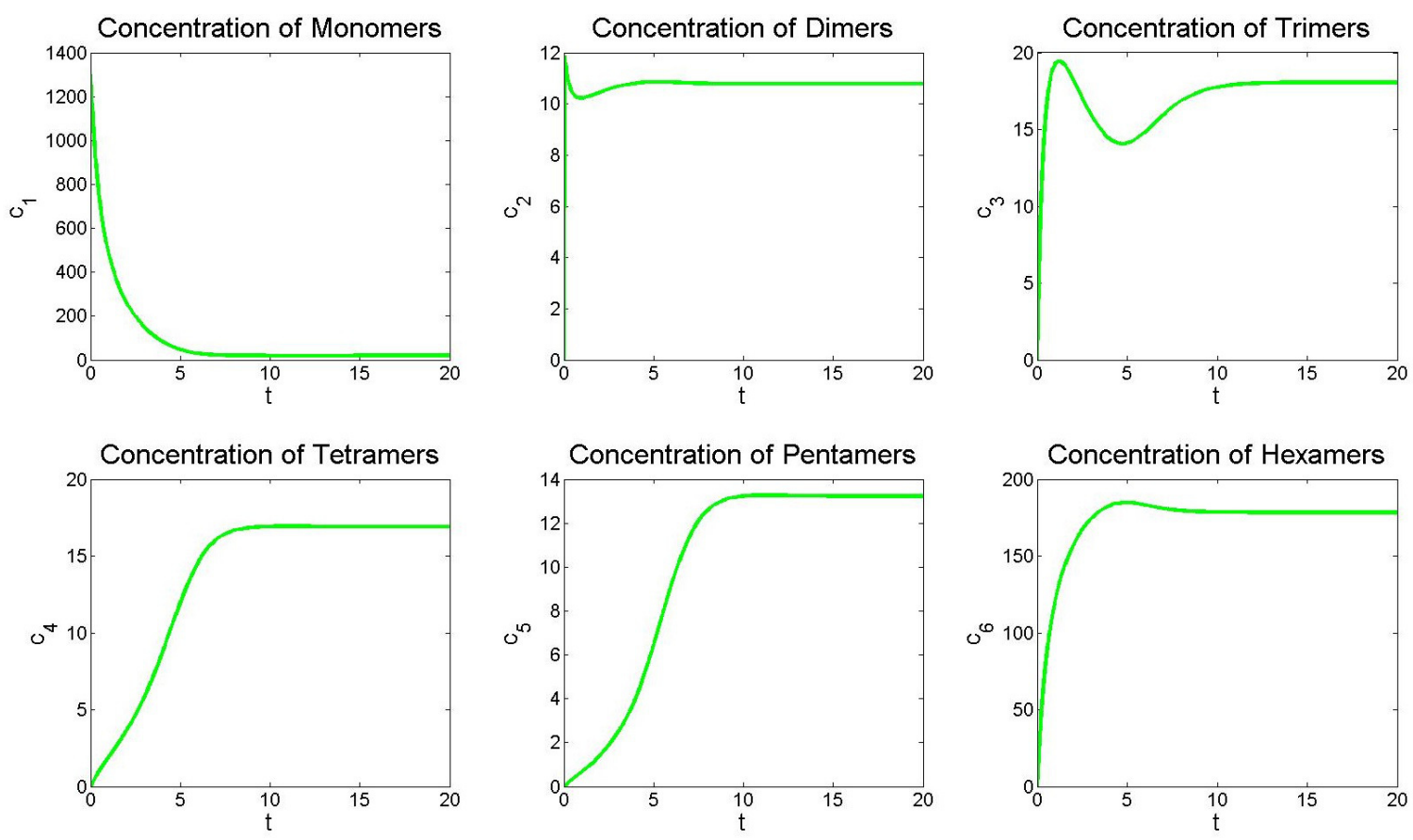

Figure 4: Simulation results: Concentrations of all intermediates $c_{n}(1 \leq n \leq 6)$ from simulation time $t=0$ to $t=20$ (second) with an initial condition $\vec{c}(0)=\left(c_{1}(0), c_{2}(0), c_{3}(0), c_{4}(0), c_{5}(0), c_{6}(0)\right)=$ $(1300,0,0,0,0,0)$. Simulations were performed until $t=24 \times 3600$ (second), though they are not shown here due to the early convergence of the solution.

first, as discussed in $[4,8,15]$. The quick decrease in $c_{2}$ indicates the importance of the dimers in building higher order n-mers. It is interesting to see the trimer concentration $c_{3}$ goes through an initial spike then a drop and then approaches the equilibrium. This will be further addressed in the subsection on embedded modeling. The concentrations $c_{n}(n=4,5,6)$ are gradually increasing as expected.

\subsection{Results of Sensitivity and Elasticity Analysis}

Sensitivity and elasticity analysis is performed for the concentration of $n$-mer $c_{n}$ $(\mathrm{n}=1,2,3,4,5,6)$ with respect to the association and dissociation rates (forward and backward rates) using the SENSAI Matlab package [38]. There are a total of 16 forward and backward rates, as shown in Figure 5.

As shown in Table 1, the model parameter values vary in three orders of magnitude. This suggests that a scaling of the parameter values is necessary and elasticity analysis may be more appropriate than just sensitivity analysis.

For the six concentrations $c_{i}(i=1, . ., 6)$ and the sixteen parameters $p_{k}(k=$ $1, \ldots, 16)$, a total of 96 derivatives need to be calculated over time. A scaling is then executed as defined in Equation (12) to obtain the elasticity. 


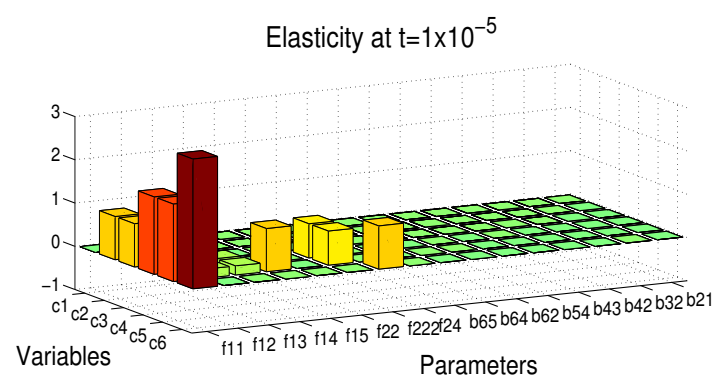

Parameters
Elasticity at $t=0.03$



Elasticity at $\mathrm{t}=1$

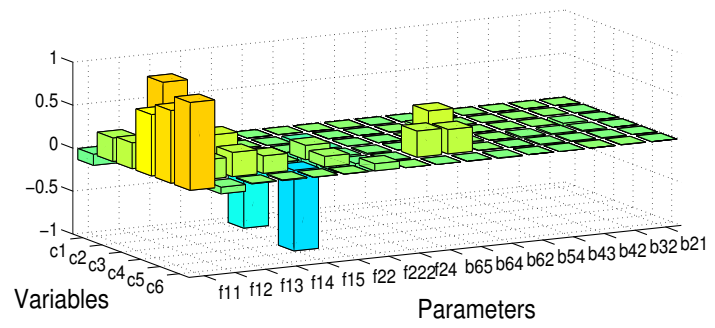



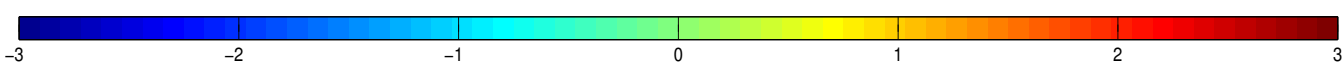

Elasticity at $\mathrm{t}=2$

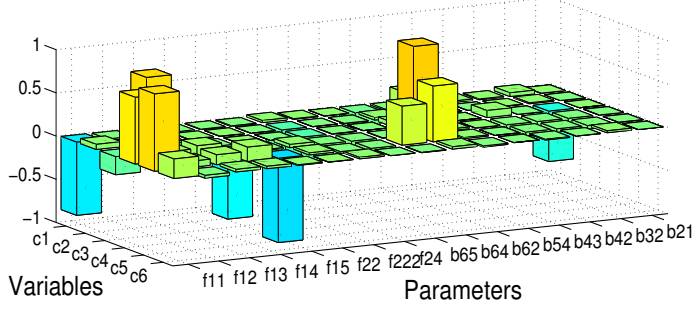

Elasticity at $\mathrm{t}=4$

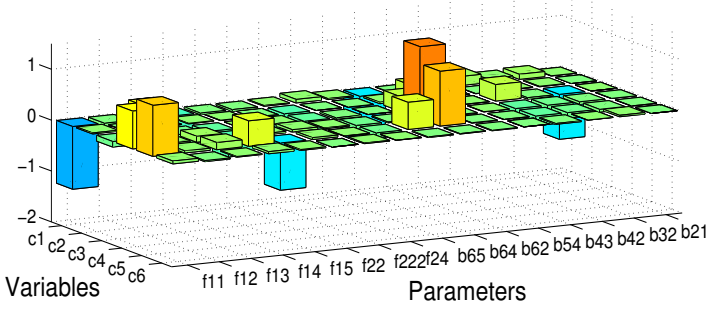

Elasticity at $\mathrm{t}=7$

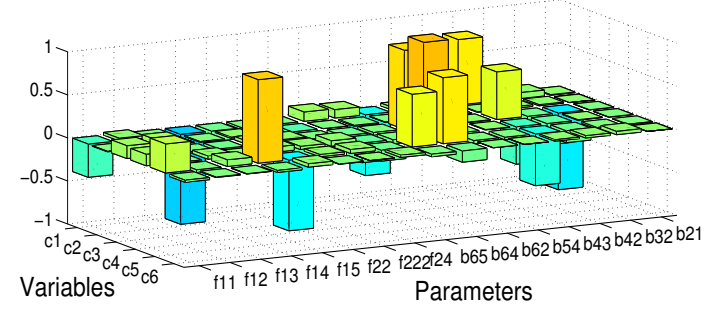

Elasticity at $\mathrm{t}=12$



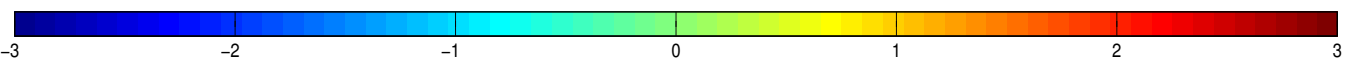

Figure 5: Elasticities of the $n$-mer concentration $c_{n}$ with respect to the association and dissociation rates are plotted for eight simulation time moments: $t=1 \times 10^{-5}, 0.03,0.1,1,2,4,7,12$ (second) 
We examine the elasticity of the concentrations to the model parameters at the following times: $t=1 \times 10^{-5}, 0.03,0.1,1,2,4,7,12$ (second). We consider the values at $t=12$ as the equilibrium values. There are rapid changes in the concentration of monomers for $t<1$ and so we consider elasticity at three other times before $t=1$, then three other times after $t=1$ but before the equilibrium.

The elasticity results tell an expected story. Near the beginning (Figure 5), concentrations are most elastic to the forward rates, especially $f_{11}$. This is intuitive, since the $c_{1}$ concentration is rapidly decreasing as the monomers are forming into dimers and trimers, as demonstrated in the spikes of $c_{2}$ and $c_{3}$ concentrations in Figure 4 . As the time increases, concentrations become less elastic to these forward rates but more elastic towards those higher intermediate forward rates, such as $f_{14}$ and $f_{15}$ (Figure 5 row 2).

There is a comparable increase in elasticity to the backward rates (Figure 5 row $3,4)$. It is interesting to note that the elasticity to parameters $b_{65}$ and $b_{64}$ appear first out of the backward rates (Figure 5 row 1 right), and remain evident throughout the rest of the simulation time period. Since hexamers are assumed to be the most stable intermediate, these results could provide information on when hexamers might disassemble.

Elasticity to the association rates $f_{1 i}, i=1, \ldots, 6$. The hexamer concentration $c_{6}$ shows the largest elasticity to the forward rate $f_{11}$ at the beginning of nucleation. Other concentrations also show elasticity to $f_{11}$ at times as expected, since $f_{11}$ is the parameter needed for nucleation to begin. These elasticities decrease as time increases, except for concentrations $c_{1}, c_{4}$, for which some fluctuations are observed. See Figure 5 (row 1,2) for $c_{1}$ and Figure 5 (row 3 right) for $c_{1}, c_{4}$. All other intermediate concentrations follow a similar pattern of decreasing in elasticity for the forward rate $f_{12}$.

The elasticity of $c_{5}$ to $f_{14}$ is seen at the beginning (Figure 5 row 1 left). It gradually increases as time goes by and the system approaches its equilibrium (Figure 5 row $3,4)$. Concentration of $c_{5}$ also shows consistent elasticity towards parameter $f_{15}$. This implies that the two forward rates $f_{14}, f_{15}$ are important for the assembly of a pentamer and hexamer. Minimal elasticity is observed for any concentration with respect to $f_{13}$.

Elasticity to the association rates $f_{22}, f_{222}, f_{24}$. Concentrations $c_{4}, c_{5}$ both demonstrate elasticity with respect to parameter $f_{22}$ at the beginning of nucleation (Figure 5 row 1). These elasticities decrease as time increases. A similar pattern is seen for $c_{6}$ with respect to $f_{222}$ as the system approaches its equilibrium. These results can be viewed as indications of the importance of the dimer intermediate in the assembly (pathways (b) and (c)).

Elasticity to the backward rates. As shown in Figure 5, the magnitude of elasticities with respect to the backward rates tends to increase whereas the magnitude 
of elasticities with respect to the forward rates decreases. Elasticity to the backward rate $b_{65}$ appears first (see Figure 5 row 1,2) and stays evident as time increases. Concentration $c_{3}$ has consistent elasticity past $t=2$ and $c_{4}$ has consistent elasticity with respect to $b_{43}$ from $t=7$ to the equilibrium. These results indicate that higher order multimers may prefer disassembly of one monomer at a time.

Concentrations $c_{4}$ and $c_{5}$ show elasticity to parameter $b_{64}$. This is expected for $c_{4}$, since the backward rate $b_{64}$ is representative of a hexamer dissociating into a tetramer and dimer. The elasticity of $c_{5}$ with respect to $b_{64}$ may be indicative of a pentamer being integrated into the lattice, as discussed in [43]. Minimal elasticity is seen for any concentration with respect to parameters $b_{62}, b_{54}, b_{42}, b_{21}$.

\subsection{Model Sensitivity and Embedded Models}

Consistent low elasticity over time could imply that certain parameters are not important for modeling capsid nucleation. These parameters may not give additional or important information for our model. To validate this claim, embedded models are analyzed to further characterize which parameters are most important for reflecting the assembly kinetics. Parameters with low elasticity are removed from the model, one at a time, to analyze its importance in the model. A parameter is deemed important only if the equilibrium solution changes or the time to equilibrium changes drastically.

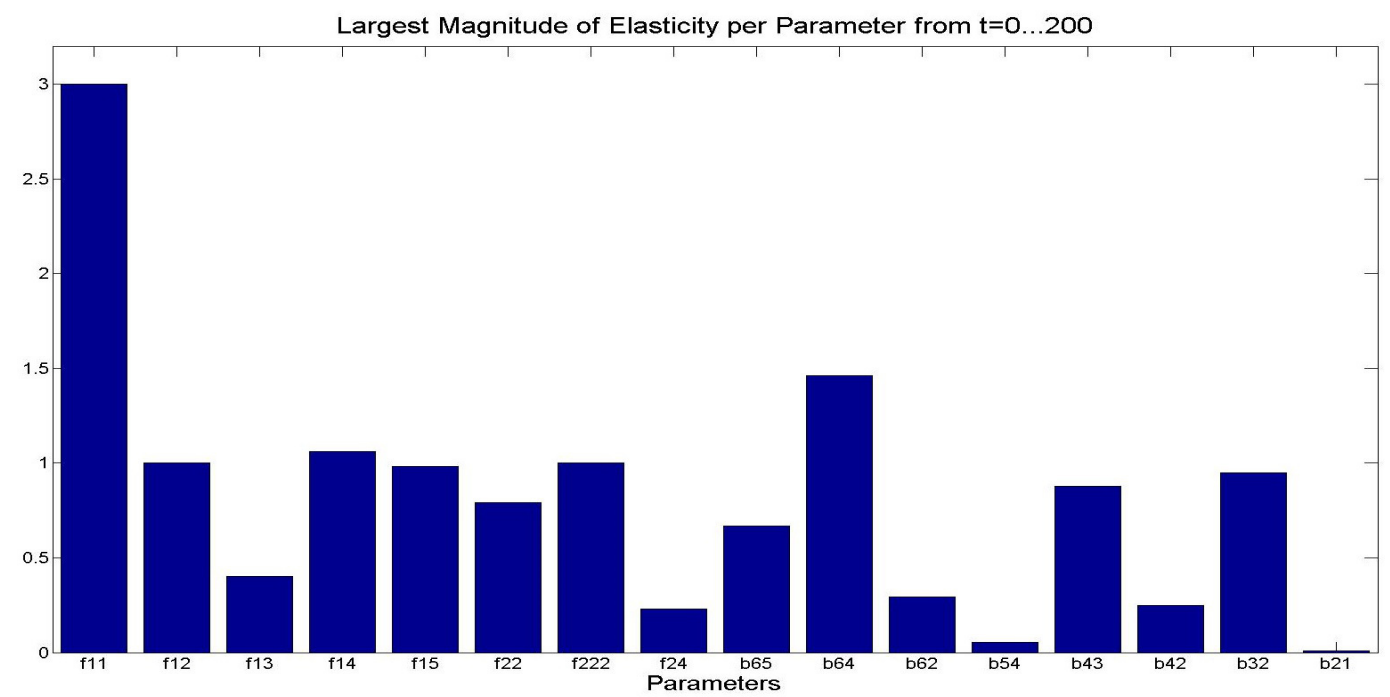

Figure 6: The largest elasticity magnitudes for the $n$-mer concentration $c_{n}$ with respect to the model parameters over all time (represented by the magnitudes of the derivative). Low elasticity is observed for parameters $f_{13}, f_{24}, b_{62}, b_{54}, b_{42}, b_{21}$. 
Table 3: Relative error by removing individual parameters

\begin{tabular}{|l|c|c|c|c|c|c|}
\hline \hline Parameters & $f_{13}$ & $f_{24}$ & $b_{62}$ & $b_{54}$ & $b_{42}$ & $b_{21}$ \\
\hline Rel. err. $\frac{\left\|X_{r}-X\right\|}{\|X\|}$ & 0.0034 & 0.0479 & 0.0314 & 0.0075 & 0.0537 & 0.0020 \\
\hline
\end{tabular}

Table 4: Relative error by removing multiple parameters simultaneously

\begin{tabular}{|l|c|c|c|c|}
\hline \hline Parameters & $f_{13}, b_{54}$ & $f_{13}, b_{21}$ & $b_{54}, b_{21}$ & $f_{13}, b_{54}, b_{21}$ \\
\hline Rel. err. $\frac{\left\|X_{r}-X\right\|}{\|X\|}$ & 0.0048 & 0.0021 & 0.0095 & 0.0068 \\
\hline
\end{tabular}

The largest magnitude of the elasticity for each concentration $c_{n}$ with respect to parameter $p_{k}$ for $0<t<200$ is shown in Figure 6 . We identify parameters with low elasticity for all concentrations $c_{n}$. The parameters of question are taken to be $f_{13}, f_{24}, b_{62}, b_{54}, b_{42}$ and $b_{21}$.

Each parameter is removed from the model, one at a time. The dynamical system is then reduced and re-solved. Equilibrium solution is evaluated and the relative error between the new equilibrium $\left(X_{r}\right)$ and the original model equilibrium $(X)$ is calculated. The results from the embedded models are listed in Table 3. It is observed that parameters $f_{13}, b_{54}, b_{21}$ can be eliminated from the model individually with negligible changes to the equilibrium concentrations.

This process is repeated by removing multiple parameters simultaneously. The relative error of removing multiple parameters are listed in Table 4 . It is clear that the three parameters $f_{13}, b_{54}, b_{21}$ can be eliminated from the model simultaneously with a negligible change to the equilibrium concentrations. By removing all three parameters, the three main pathways for assembly of a hexamer change. The new pathways are listed below.

(a') Single monomers join (reduced):

$$
c_{1}+c_{1} \stackrel{f_{11}}{\longrightarrow} c_{2}, \quad c_{1}+c_{2} \underset{b_{32}}{\stackrel{f_{12}}{\rightleftharpoons}} c_{3}, \quad c_{1}+c_{3} \underset{b_{43}}{\tau} c_{4}, \quad c_{1}+c_{4} \stackrel{f_{14}}{\longrightarrow} c_{5}, \quad c_{1}+c_{5} \underset{b_{65}}{\stackrel{f_{15}}{\rightleftharpoons}} c_{6} .
$$

(b') Trimer-of-dimers (reduced):

$$
c_{1}+c_{1} \stackrel{f_{11}}{\longrightarrow} c_{2}, \quad c_{2}+c_{2}+c_{2} \underset{b_{62}}{\stackrel{f_{222}}{\rightleftharpoons}} c_{6}
$$

(c') Single binding dimers (reduced):

$$
c_{1}+c_{1} \stackrel{f_{11}}{\longrightarrow} c_{2}, \quad c_{2}+c_{2} \underset{b_{42}}{\stackrel{f_{22}}{\rightleftharpoons}} c_{4}, \quad c_{2}+c_{4} \underset{b_{64}}{\stackrel{f_{24}}{\rightleftharpoons}} c_{6}
$$


By removing parameters $b_{54}, b_{21}$, pentamers and dimers are no longer able to dissociate in the new model. Similarly, by removing parameter $f_{13}$, there is only one pathway for tetramer assembly (pathway (c'), two dimers forming a tetramer). It is interesting to note that all three of these parameters are found in the traditional pathway (a), as discussed in the studies presented in [18, 45]. Removal of these parameters disrupts this pathway.

Calculating the probability of each pathway would be helpful for identifying the usefulness of the traditional pathway in the existing work, compared to the two new pathways for hexamer assembly investigated in this paper: single binding dimers (pathway (c)) and the trimer-of-dimer (pathway (b)).

\subsection{Full Model vs Reduced Model}

In Subsection 2.1, we proposed a full model for HIV-1 capsid nucleation by considering theoretically possible pathways. A reduced model is derived in Subsection 2.2 by eliminating certain pathways based on biological evidence in the literature that these pathways are less likely. In Subsections 4.2-4, we conducted numerical simulations as well as sensitivity and elasticity analysis to examine which parameters in the reduced model are less significant. Then further reductions of the reduced model were examined to verify that indeed these further reduced models (or embedded models) can still catch the main features of the association and dissociation processes.

The aforementioned parameter fitting and model reduction methodology can also be applied directly to the full model proposed in Subsection 1.1.

Table 5: Comparison of fitted values for the parameters in the full and reduced models

\begin{tabular}{||r|r|r||r|r|r||r|r|r|}
\hline \hline & Full & Reduced & & Full & Reduced & & Full & Reduced \\
\hline$f_{11}$ & 0.000498 & 0.000556 & $b_{65}$ & 0.205719 & 0.193838 & $f_{23}$ & 0.001434 & N/A \\
$f_{12}$ & 0.004585 & 0.004506 & $b_{64}$ & 0.263029 & 0.256905 & $f_{33}$ & 0.001092 & N/A \\
$f_{13}$ & 0.000830 & 0.000867 & $b_{62}$ & 0.960900 & 0.993826 & $b_{63}$ & 0.012523 & N/A \\
$f_{14}$ & 0.040147 & 0.038226 & $b_{54}$ & 0.109395 & 0.056015 & $b_{53}$ & 0.004154 & N/A \\
$f_{15}$ & 0.169364 & 0.179675 & $b_{43}$ & 0.556444 & 0.728455 & & & \\
$f_{22}$ & 0.013115 & 0.013196 & $b_{42}$ & 0.738419 & 0.719905 & & & \\
$f_{222}$ & 0.161355 & 0.159765 & $b_{32}$ & 0.685344 & 0.717905 & & & \\
$f_{24}$ & 0.106903 & 0.061905 & $b_{21}$ & 0.028071 & 0.019094 & & & \\
\hline
\end{tabular}

The full model (Equation (1)) has 20 parameters, whereas the reduced model (Equation (2)) has 16 parameters. Parameter fitting was applied to the reduced model and the fitted values were listed in Table 1. Parameter fitting is now applied to the full model and the fitted values are listed in Table 5, along with the values from Table 1. It can be observed from Table 5 that for the 16 parameters retained 
in the reduced model, their numerical values in these two rounds of fitting are very close.
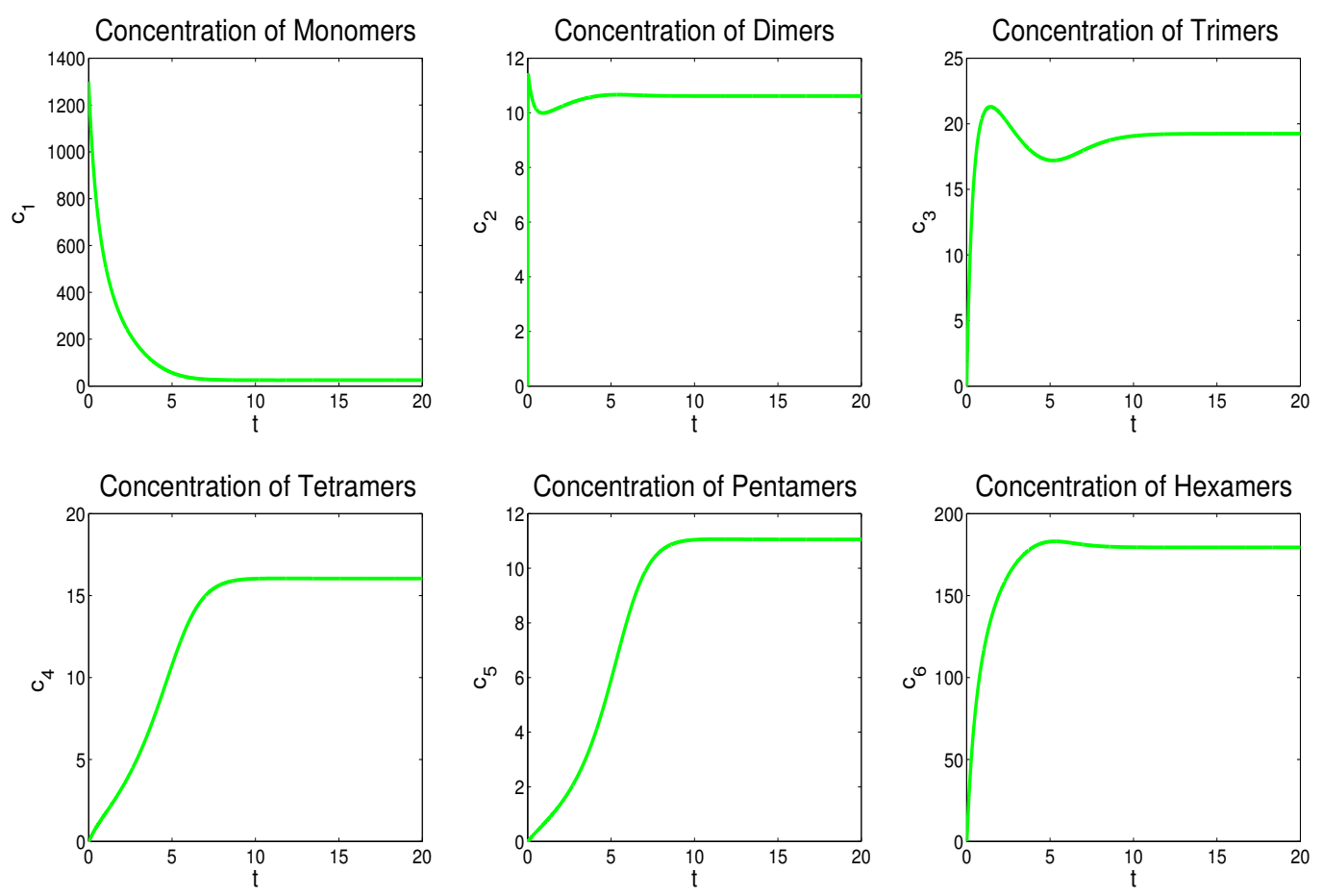

Figure 7: Simulation results for the full model in Equation (1): Concentrations of all intermediates $c_{n}(1 \leq n \leq 6)$ from simulation time $t=0$ to $t=20$ (second) with an initial condition $\vec{c}(0)=$ $\left(c_{1}(0), c_{2}(0), c_{3}(0), c_{4}(0), c_{5}(0), c_{6}(0)\right)=(1300,0,0,0,0,0)$. Simulations were performed until $t=$ $24 \times 3600$ (second), though they are not shown here due to the early convergence of the solution. These results are very similar to those shown in Figure 4.

For the full model with the fitted values of these 20 parameters, we perform also numerical simulations and plot the multimer concentrations $\left(c_{1}\right.$ through $\left.c_{6}\right)$ in Figure 7. It can be observed from Figures 4 and 7 that these concentration profiles are very similar for the full model and the reduced model.

Furthermore, we perform elasticity analysis for the 20 parameters in the full model, in the same way as we did for the reduced model. As shown in Figure 8, the parameters $f_{23}, f_{33}, b_{53}$ have clearly very small magnitude in elasticity. These three parameters are among the four parameters $f_{23}, f_{33}, b_{63}, b_{53}$, which are in the reduction (from the full model to the reduced model) investigated in Subsection 1.1 and 1.2. 




Figure 8: The largest elasticity magnitudes for the 20 parameters of the full model (Equation (1)).

\section{Discussion}

This paper focuses on the nucleation stage of viral capsid assembly. It is different than the existing work $[12,18,26]$ that consider mainly one pathway and add/delete one capsomer unit at a time. Our model considers more pathways for association and dissociation and provides more information about the assembly. It is now revealed by the model that CA dimers indeed play an important role in the nucleation stage, as reflected in two results: (i) the initial spike in the dimer concentrations in the numerical simulations; (ii) analysis showing that $f_{22}, f_{24}, f_{222}$ are important parameters for HIV-1 capsid nucleation. These results conform with the findings in $[4,8,15,40]$.

Parameters $f_{11}, f_{12}, b_{64}$ exhibit elasticity in the monomer and hexamer concentrations $c_{1}, c_{6}$. These three association or dissociation rates correspond respectively to three reactions: (i) two monomers forming a dimer; (ii) a monomer and dimer together producing a trimer; (iii) a hexamer breaking apart into a tetramer and dimer. Examination of elasticity at different times helps determine which pathway is the most important. For instance, after the initial spike of the concentration of dimers, the concentrations of the intermediates become more sensitive to $f_{222}$. This is an indication of the importance of three dimers forming a hexamer. These results imply that the most important pathways for hexamer formation are single monomers joining together and triple binding dimers. These results demonstrate that our model has predictability to a certain level.

This paper applies also sensitivity and elasticity analysis for model reduction by identifying insignificant or less important model parameters. The reduced model is validated by agreement of biological experiment data and in silicon results. In general, an alternation or perturbation of a dynamical system will result in the fundamental 
issue of global stability and/or bi-stability [32]. New mathematical tools like those in [39] need to be developed to address the global stability of the polynomial autonomous dynamical systems for viral capsid nucleation.

Clearly, there exists randomness in the nucleation stage of viral capsid assembly. The temperature, pH-value, and many other factors in the environment of assembly affect the association and dissociation rates and hence the formation of $\mathrm{CA}$ hexameters and pentamers. Our future work includes investigation of the stochastic features of nucleation and stochastic dynamical systems will be an indispensable tool [2].

The investigation of nucleation cannot be completely isolated from the whole process of viral capsid assembly. It is our postulation that at the early stage of viral capsid assembly, hexamer formation happens simultaneously in many locations within the virion. Then these hexamers further assemble into the viral capsid. Pentamers might form at the places where it is difficult for a hexamer to form. This is the elongation stage. In other words, the products of nucleation serves as feed of the elongation stage. We foresee a cascade of kinetics and cascaded stochastic dynamical systems (CSDS) shall be an exploratory tool for this investigation.

Acknowledgments: Farrah Sadre-Marandi was partially supported by US National Science Foundation under grant IIA-141511 and Colorado State University Yates Graduate Fellowship. Yuewu Liu and Xiufen Zou were partially supported by the Major Research Plan of the National Natural Science Foundation of China (No.91230118) and the National Natural Science Foundation of China (No.61173060). The 1st, 3rd, 4th would like to express their sincere thanks to Prof. Chaoping Chen

of Department of Biochemistry and Molecular Biology at Colorado State University for her great help and the stimulating discussion.

\section{References}

[1] A. Alfadhli, D. Huseby, E. Kapit, D. Colman, E. Barklis, Human immunodeficiency virus type 1 matrix protein assembles on membranes as a hexamer, J. Virol., 81(2006), pp. 1472-1478.

[2] J.E. Baschek, H. Klein, U.S. Schwarz, Stochastic dynamics of virus capsid formation: direct versus hierarchical self-assembly, BMC Biophys., 5(2012), pp. 1-18

[3] J. Benjamin, B.K. Ganser-Pornillos, W.F. Tivol, W.I. Sundquist, G.J. Jensen, Three-dimensional structure of HIV-1 virus-like particles by electron cryotomography, J. Mol. Biol., 346(2005), pp. 577-588.

[4] L. Briant, B. Gay, C. Devaux, N. Chazal, HIV-1 assembly, release, and maturation, World J. of AIDS, 1(2011), pp. 111-130. 
[5] J. Briggs, K. Grünewald, B. Glass, F. Förster, H.-G. Kräusslich, S.D. Fuller, The mechanism of HIV-1 core assembly: Insights from three-dimensional reconstructions of authentic virions, Structure, 14(2006), pp. 15-20.

[6] J. Briggs, H.G. Kräusslich, The molecular architecture of HIV, J. Mol. Biol., 410(2011), pp. 491-500.

[7] J. Briggs, J.D. Riches, B. Galss, V. Bartonova, G. Zanetti, H.-G. Kräusslich, Structure and assembly of immature HIV, PNAS, 106(2009), pp. 11090-11095.

[8] I.L. Byeon, X. Meng, J. Jung, G. Zhao, R. Yang, J. Ahn, J. Shi, J. Concel, C. Aiken, P. Zhang, A.M. Gronenborn, Structural convergence between cryo-EM and NMR reveals intersubunit interactions critical for HIV-1 capsid formation, Cell, 139(2009), pp. 780-790.

[9] D. Caspar, A. Klug, Physical principles in the construction of regular viruses, Cold Spring Harb. Symp. Quant. Biol., 27(1962), pp. 1-24.

[10] B. Chen, R. Tycko, Simulated self-assembly of the HIV-1 capsid: Protein shape and native contacts are sufficient for two-dimensional lattice formation, Biophys. J., 100(2011), pp. 3035-3044.

[11] R.C. Eberhart, J. Kennedyäusslich, A new optimizer using particle swarm theory, Proc. 6th Intl. Symp. Micro Machine \& Human Sci., 1(2011), pp. 39-43.

[12] D. Endres, A. Zlotnick, Model-based analysis of assembly kinetics for virus capsids or other spherical polymers, Biophys. J., 83(2002), pp. 1217-1230.

[13] B. Ganser-Pornillos, A. Cheng, M. Yeager, Structure of full-length HIV-1 CA: A model for the mature capsid lattice, Cell, 131(2007), pp. 70-79.

[14] B. Ganser-Pornillos, M. Yeager, O. Pornillos, Assembly and architecture of HIV, Viral Molecular Machines (Book Chapter), 726(2008), pp. 441-465.

[15] B. Ganser-Pornillos, M. Yeager, W.I. Sundquist, The structural biology of HIV assembly, Structural Biology, 18(2008), pp. 203-217.

[16] J. Grime, G.A. Voth, Early stages of the HIV-1 capsid protein lattice formation, Biophys. J., 103(2012), pp. 1774-1783.

[17] M. Hagan, Modeling Viral Capsid Assembly, Adv. Chem. Phys., 155(2014), pp. 1-34.

[18] M. Hagan, O. Elrad, Understanding the concentration dependence of viral capsid assembly kinetics - the origin of the lag time and identifying the critical nucleus size, Biophys. J, 98(2010), pp. 1065-1074. 
[19] S. Jin, L. Niu, G. Wang, X. Zou, Mathematical modeling and nonlinear dynamical analysis of cell growth in response to antibiotics, Intl. J. Bifur. Chaos, 25(2015), DOI:10.1142/S0218127415400076

[20] S. Katen, A. Zlotnick, The thermodynamics of virus capsid assembly, Meth. Enzymol., 455(2009), pp. 395-417.

[21] N. Komarova, D. Levy, D. Wodarz, Effect of synaptic transmission on viral fitness in HIV infection, PLoS ONE, 7(2012), e48361.

[22] J. Lanman, T.T. Lam, S. Barnes, M. Sakalian, M.R. Emmett, A.G Marshall, P.E. Preveige, Identification of novel interactions in HIV-1 capsid protein assembly by high-resolution mass spectrometry, J. Mol. Biol., 325(2003), pp. 759-772.

[23] S. Li, C.P. Hill, W.I. Sundquist, J.T. Finch, Image reconstructions of helical assemblies of the HIV-1 CA protein, Nature, 407(2000), pp. 409-413.

[24] S. Liu, L. Pang, S. Ruan, X. Zhang, Global dynamics of avian influenza epidemic models with psychological effect, Comput. Math. Meth. Med., Vol. 2015, Article ID 913726.

[25] K. Mayo, D. Huseby, J. McDermott, B. Arvidson, L. Finlay, E. Barklis, Retrovirus capsid protein assembly arrangements, J. Mol. Biol., 325(2003), pp. 225237.

[26] R. Munoz-Alicea, HIV-1 gag trafficking and assembly: Mathematical models and numerical simulations, Ph.D. Dissertation, Colorado State University, 2013.

[27] O. Pornillos, B.K. Ganser-Pornillos, M. Yeager, Atomic-level modelling of the HIV capsid, Nature, 469(2011), pp. 424-428.

[28] O. Pornillos, B. Ganser-Pornillos, M. Yeager, Disulfide bond stabilization of the hexameric capsomer of human immunodeficiency virus, J. Mol. Biol., 401(2010), pp. 985-995.

[29] Protein Data Bank, http://www.rcsb.org/pdb/home/home.do

[30] O. Pornillos, B. Ganser-Pornillos, B. Kelly, Y. Hua, F. Whitby, C. Stout, W. Sundquist, C. Hill, M. Yeager, X-ray structures of the hexameric building block of the HIV capsid, Cell, 137(2009), pp. 1282-1292.

[31] L. Roeger, Z. Feng, C. Castillo-Chavez Modeling TB and HIV co-infections, Math. Biosci. Eng., 6(2009), pp. 815-837.

[32] M. Shub, Global stability of dynamical systems, Springer, 1987. 
[33] J.K. Stille, Step-growth polymerization, J. Chem. Ed., 58(1981), pp. 862-866.

[34] W. Sundquist, H. Kräusslich, HIV-1 assembly, budding, and maturation, Cold Spring Harbor Perspect Med., 2(2012), pp. 1-24.

[35] J. Tan, X. Zou, Optimal control strategy for abnormal innate immune response, Comput. Math. Meth. Med., (2015), Article ID 386235, DOI:10.1155/2015/386235

[36] J. Tan, X. Zou, Complex dynamical analysis of a coupled system from innate immune responses, Intl. J. Bifur. Chaos, 23(2013),

[37] S. Tavener, M. Mikucki, S. Field, M.F. Antolin, Transient sensitivity analysis for nonlinear population models, Meth. Eco. Evol., 2(2011), pp. 560-575.

[38] S. Tavener, M. Mikucki, SENSAI: A Matlab package for sensitivity analysis, http://www . math. colostate.edu/ tavener/FEScUE/SENSAI/sensai.shtml

[39] J.P. Tian, J. Wang, Global stability for cholera epidemic models, Math. Biosci., 232(2011), pp. 31-41.

[40] B.G. Turner, M.F. Summers, Structural Biology of HIV, J. Mol. Biol., 285(1999), pp. 1-32.

[41] Y. Wang, J. Tan, F. Sadre-Marandi, J. Liu, X. Zou, Mathematical modeling for intracellular transport and binding of HIV-1 gag proteins, Math. Biosci., 262(2015), pp. 198-205.

[42] D. Wodarz, Mathematical models of HIV replication and pathogenesis, Meth. Mol. Biol., 1184(2014), pp. 563-581.

[43] M. Yeager, Design of in vitro symmetric complexes and analysis by hybrid methods reveal mechanisms of HIV capsid assembly, J. Mol. Biol., 410(2011), pp. 534552 .

[44] G. Zhao, J. Perilla, E. Yufenyuy, X. Meng, B. Chen, J. Ning, J. Ahn, A. Gronenborn, K. Schulten, C. Aiken, and P. Zhang, Mature HIV-1 capsid structure by cryo-electron microscopy and all-atom molecular dynamics, Nature (Letter), 497(2013), pp. 642-646.

[45] A. Zlotnick, J.M. Johnson, P.W. Wingfield, S.J. Stahl, D. Endres, A theoretical model successfully identifies features of hepatitis b virus capsid assembly, Biochem., 38(1999), pp. 14644-14652. 